\title{
Modification de l'hygiène de vie pour traiter la fibrillation auriculaire
}

\author{
Yehia Fanous MD, Paul Dorian MD
}

Citation : CMAJ 2020 November 16;192:E1469. doi : 10.1503/cmaj.201084-f

Voir la version anglaise de l'article ici : www.cmaj.ca/lookup/doi/10.1503/cmaj.201084

\section{La perte de poids obtenue et maintenue à l'aide d'une diète et de \\ 1 l'exercice physique est associée à un nombre réduit d'épisodes de fibrillation auriculaire $(F A)^{1}$}

Les essais qui ont fait état des bienfaits de la perte de poids (associée à d'autres stratégies de prise en charge des facteurs de risque) dans les cas de fibrillation auriculaire ont principalement eu recours à des diètes à forte teneur en protéines et à faible indice glycémique, combinées à des exercices de faible intensité, afin de faire diminuer l'indice de masse corporelle (IMC) $)^{1,2}$. Les patients atteints de FA intermittente et ayant un IMC $\geq 27 \mathrm{~kg} / \mathrm{m}^{2}$ ont connu l'augmentation la plus marquée de la survie sans arythmie avec le maintien d'une perte de poids corporel d'au moins $10 \%$ (nombre de sujets à traiter $[\mathrm{NST}]=4,8)^{1-3}$.

2 La pratique d'activités physiques récréatives confère une cardioprotection aux patients atteints de $F A$, indépendamment de la perte de poids $s^{1-3}$ Des études d'observation montrent que, quelle qu'en soit l'intensité, l'exercice fait de façon récréative est associé à un risque moindre de développer une FA et à une meilleure maîtrise des symptômes chez les patients qui ont une FA persistante ${ }^{1,3,4}$. Une bonne cible est d'atteindre progressivement les 200 minutes d'exercice d'intensité modérée par semaine (1000 équivalents métaboliques [MET]), durée qui entraîne une baisse d'environ $10 \%$ du risque de FA incidente ${ }^{1,3}$.

La réduction de la consommation d'alcool chez les personnes qui prennent plus de 14 consommations par semaine est associée à une diminution des épisodes de $F A$ et des hospitalisations pour cause de $F A^{13,5}$

Les données sont contradictoires : certaines indiquent que le risque de FA est en corrélation linéaire avec la quantité consommée, et d'autres qu'il existe un seuil au-delà duquel le risque augmente ${ }^{1,3}$. Un essai randomisé contrôlé récent a montré que l'abstinence réduisait les épisodes (NST $=5$ ) chez les consommateurs modérés (17 \pm 8 consommations/semaine) atteints de FA intermittente ${ }^{1}$. Il faut conseiller aux buveurs modérés et aux grands buveurs, et surtout à ceux qui s'adonnent au calage, de réduire leur consommation.

4.

L'abandon du tabagisme fait partie des stratégies recommandées pour prévenir et contrôler la FA ${ }^{1}$

On pense qu'il existe un lien dose-dépendant entre le tabagisme et la FA ${ }^{1}$. En outre, le tabagisme est étroitement associé à la maladie pulmonaire obstructive chronique, un important facteur de risque de $\mathrm{FA}^{1}$.

\section{Limiter la consommation de caféine a peu de chances de réduire \\ 3 l'incidence ou les effets de la $F A^{1,3}$}

Aucune donnée probante ne permet de conclure qu'abaisser sa consommation habituelle de caféine réduirait le risque de $F A^{1}$.

\section{Références}

1. Chung MK, Eckhardt LL, Chen LY, et al.; American Heart Association Electrocardiography and Arrhythmias Committee and Exercise, Cardiac Rehabilitation, and Secondary Prevention Committee of the Council on Clinical Cardiology; Council on Arteriosclerosis, Thrombosis and Vascular Biology; Council on Cardiovascular and Stroke Nursing; Council on Lifestyle and Cardiometabolic Health. Lifestyle and risk factor modification for reduction of atrial fibrillation: a scientific statement from the American Heart Association. Circulation 2020;141:e750-72.

2. Pathak RK, Middeldorp ME, Meredith M, et al. Longterm effect of goal-directed weight management in an atrial fibrillation cohort: a long-term follow-up study (LEGACY). J Am Coll Cardiol 2015;65:2159-69.

3. Wingerter R, Steiger N, Burrows A, et al. Impact of lifestyle modification on atrial fibrillation. $A m ~ J$ Cardiol 2020;125:289-97.

4. Svedberg N, Sundström J, James S, et al. Long-term incidence of atrial fibrillation and stroke among cross-country skiers. Circulation 2019;140:910-20.

5. Gémes K, Malmo V, Laugsand LE, et al. Does moderate drinking increase the risk of atrial fibrillation? The Norwegian HUNT (Nord-Trøndelag Health) study. J Am Heart Assoc 2017;6:e007094.

Intérêts concurrents : Aucun déclaré.

Cet article a été révisé par des pairs.

Affiliations : Département de médecine (Fanous), Université Western Ontario, London, Ont.; Division de cardiologie (Dorian), Service de médecine, Hôpital St. Michael; Faculté de médecine (Dorian), Université de Toronto, Toronto, Ont.

Propriété intellectuelle du contenu : II s'agit d'un article en libre accès distribué conformément aux modalités de la licence Creative Commons Attribution (CC BY-NCND 4.0), qui permet l'utilisation, la diffusion et la reproduction dans tout médium à la condition que la publication originale soit adéquatement citée, que l'utilisation se fasse à des fins non commerciales (c.-à-d., recherche ou éducation) et qu'aucune modification ni adaptation n'y soit apportée. Voir : https://creativecommons.org/ licenses/by-nc-nd/4.0/

Correspondance : Paul Dorian, pauldorian@unityhealth.to 